\title{
Simulations and measurements of the impact of collective effects on dynamic aperture
}

\author{
Vadim Sajaev, Ryan Lindberg, ${ }^{*}$ and Michael Borland \\ Advanced Photon Source, Argonne National Laboratory, Argonne, Illinois 60439, USA \\ Seunghwan Shin ${ }^{\dagger}$ \\ Pohang Accelerator Laboratory, POSTECH, Pohang, Kyungbuk 790-784, Korea
}

(Received 18 September 2018; published 19 March 2019)

\begin{abstract}
We describe a benchmark study of collective and nonlinear dynamics in the Advanced Photon Source storage ring. A 1-mm long bunch was assumed in the calculation of wakefield and element by element particle tracking with distributed wakefield components along the ring was performed in an ELEGANT simulation. Although the accurate prediction of nonlinear beam dynamics with collective effects is extremely challenging, the simulations agree well with experimental measurements.
\end{abstract}

DOI: 10.1103/PhysRevAccelBeams.22.032802

\section{INTRODUCTION}

Storage rings based on the multibend achromat (MBA) lattice concept [1] are emerging as part of a worldwide push beyond the brightness and coherence reached by present third-generation storage rings. MAX-IV [2] was the first MBA machine to be built and is now in operation. It will be followed shortly by SIRIUS [3], which is currently under construction. Other projects are underway to convert an existing, third-generation storage rings such as ESRF, APS, Spring-8, and ALS [4-7] into a fourth-generation storage rings (4GSR). In the upgrade lattice designs for these facilities, the beam emittance is pushed down to a few hundred picometers, or even $\leq 100 \mathrm{pm}$.

When the emittance of storage ring is pushed to an extremely small value, such as the $42 \mathrm{pm}$ achieved by the APS Upgrade (APS-U) design [8], two primary consequences emerge: first, the dynamic aperture typically becomes insufficient to enable accumulation based injection, so only on-axis swap-out injection $[9,10]$ is workable. Second, the maximum single bunch intensity is significantly reduced due to intrabeam scattering (IBS) and beam instabilities. Recent simulations [11] indicate that collective effects at injection are a critical issue when striving to achieve high single-bunch charge. For example, in a previously considered alternative design to the APS-U, simulations showed that collective effects at injection

\footnotetext{
*lindberg@anl.gov

tlssh@postech.ac.kr
}

Published by the American Physical Society under the terms of the Creative Commons Attribution 4.0 International license. Further distribution of this work must maintain attribution to the author(s) and the published article's title, journal citation, and DOI. precluded accumulation of the $4.2 \mathrm{~mA} /$ bunch desired for timing mode [12]. This, in addition to the desire for lower emittance, was a primary reason that the APS-U design abandoned accumulation in favor of swap-out.

Beam loss at high charge during injection into a 4GSR is a critical issue for operating these challenging future facilities, so careful modeling, simulation, and benchmarking studies must be conducted to validate the methods used in modeling collective effects. The existing APS storage ring and its impedance model provide the opportunity to perform such studies. As a first step in this process, the existing impedance database $[13,14]$ for the APS storage ring was updated to reflect recent changes to the vacuum system. This is described in more detail in [11] and below. As reported in [11], using this impedance model, simulations were performed with ELEGANT [15] and compared to new measurements. The predicted bunch length and energy spread vs bunch charge were found in good agreement with measurements, as was the accumulation limit. Other efforts to validate the APS impedance model have shown similarly good agreement. For example, in [16] we measured the effect of lattice function changes at the insertion devices and found that it agreed with predictions. This level of agreement was achieved by first carefully identifying all relevant sources of impedance and then by refining our techniques to numerically calculate both the wakefields and particle dynamics in tracking.

In this paper, we extend these studies with a detailed investigation of the stability of high-charge bunches when given large transverse kicks, which is relevant to accumulation of high-charge bunches when the dynamic acceptance (DA) is small, requiring sharing of the dynamic aperture between the stored and injected beam. Rather than characterize this in terms of the accumulation limit, we look 
at a more fundamental quantity, the inferred DA. This is determined in terms of the fraction of the stored bunch that is retained after kicking the bunch, as a function of the kick amplitude. Section II provides more detail on the construction of the impedance model for APS, then describes the methods used to track particles with collective effects. Section III describes the results of the simulations. Section IV compares measurement and simulation, and also provides an intuitive explanation of the beam dynamics. Finally, Sec. V presents conclusions.

\section{IMPEDANCE MODEL FOR APS}

The effects of impedances (wakefields) may be modeled using a number of discrete impedance elements distributed throughout the lattice. As discussed in [11], in many simulations it is sufficient to include one impedance element in the ring for simulating wakefield effects, in part because the synchrotron frequency is much longer than the revolution frequency. If the betatron tune approaches an integer, however, then modeling the ring-distributed impedance by a single element applied once per turn is no longer a good approximation, because all the simulated kicks add coherently in a way that is quite different from the actual system. Since we are simulating the behavior of the beam near the stability boundary we sought to avoid this problem by dividing the impedance into 40 identical elements that were applied in every straight section of the simulated APS. It is reasonable to locate the impedance elements in the straight sections since the majority of the effects come from the insertion device chambers. Additionally, given that we must use element-by-element tracking in these simulations (see the next section), the use of multiple impedance elements does not result in a large increase in simulation run time.

The impedance model is developed by separately considering its resistive and geometric components. The resistive wall impedance is computed using analytic formulas for elliptical chambers, which is approximately the same as that for two flat plates for the 2:1 aspect ratio of the APS geometry [17]. In contrast, because the geometric impedance depends on the detailed cross-sectional variation of various components along the vacuum vessel, the geometric contribution must be built up from the individual wakefields generated by various elements around the ring. The wakefields are calculated using the fully 3-D finite-difference time-domain code GdfidL [18]. To balance numerical efficiency and accuracy, we model the pointparticle wakefields by using the wake potential generated by a 1-mm-long Gaussian bunch in GdfidL; this approximation has had success in predicting the onset of various instabilities in the current APS, as described in $[11,13,14]$. In addition, numerical tests have been performed using wake potentials derived from electron bunches $<1 \mathrm{~mm}$ long, and these have proven to give the same longitudinal bunch lengthening and microwave instability limit as well as the same single-bunch current limit. Since simulations with more frequency bins require proportionally more particles, we use the impedance from the $1 \mathrm{~mm}$ bunch for all results here.

To understand why using a 1-mm-long Gaussian bunch is workable, one should first recognize that the impedance generated by a numerical code that employs a Gaussian bunch of width $\sigma_{b}$ is equivalent to applying a Gaussian filter of frequency width $\sigma_{f}=c / 2 \pi \sigma_{b} \approx 50 \mathrm{GHz}$ to the "true impedance." Our choice of $\sigma_{f} \approx 50 \mathrm{GHz}$ is much higher than the frequency spectrum of the zero-current APS bunch (whose length is $6 \mathrm{~mm}$ ), and also is sufficiently high to resolve the relevant impedance features of the APS. In particular, Fig. 1 shows that we resolve the first major resonancelike peak in $Z_{z}(f)$ that occurs around $20 \mathrm{GHz}$ and is the main driver of the microwave instability. Furthermore, the filter width is much larger than the maximum chromatic frequency shift, which is required to predict transverse collective effects; for the present results with $\xi=6$, this chromatic frequency shift is about $6 \mathrm{GHz}$. Of course, the wakefields from a Gaussian bunch are not strictly causal, but we believe that this is a reasonable sacrifice to make in order to have a wellunderstood and controlled approximate prescription for calculating the impedance.

Once we have computed the wakefields of all components, the total transverse wake potential of the ring is found by weighting the individual geometric contributions by their respective local lattice functions, then summing. More precisely, if each element is indexed by $j$ and the lattice functions at that element are represented by $\beta_{x, j}$ and $\beta_{y, j}$, the weighted geometric wakefields along the $x$ direction is given by

$$
\left\langle\beta_{x} W_{x}^{\mathrm{geo}}\right\rangle=\sum_{\text {elements } j} \beta_{x, j} W_{x, j}^{\mathrm{geo}},
$$

with a similar expression in $y$. The total longitudinal wakefield is given by the simple sum

$$
\left\langle W_{z}^{\mathrm{geo}}\right\rangle=\sum_{\text {elements } j} W_{z, j}^{\mathrm{geo}} .
$$

The corresponding geometric impedances are then computed using the discrete Fourier transform, and the total impedance is obtained by adding the resistive wall contribution. Finally, each impedance element is applied in the particle tracking code ELEGANT by dividing by the lattice function at its chosen location.

In a two-particle model, the longitudinal wakefield changes the test particle energy according to

$$
\Delta \gamma_{\mathrm{test}} \approx-\frac{e}{m c^{2}} W_{z}\left(z_{\mathrm{drive}}-z_{\mathrm{test}}\right)
$$

where $z_{\text {drive }}$ is the location of the drive particle, $z_{\text {test }}$ is the location of the trailing particle, and $W_{z}$ is the longitudinal 

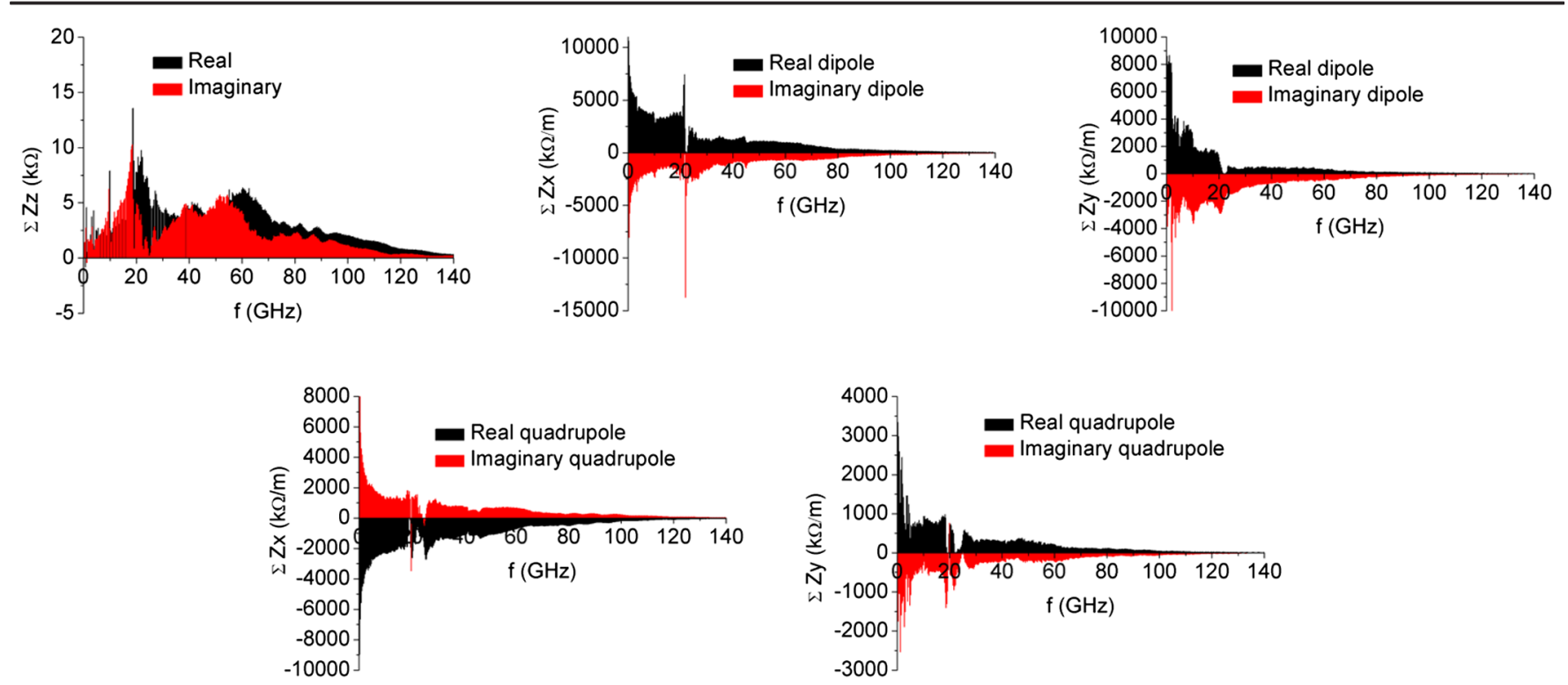

FIG. 1. APS impedance; longitudinal impedance (z), transverse ( $x$ and $y$ ) dipole impedance, and transverse $(x$ and $y)$ quadrupole impedance.

wake function. Similarly, the transverse wakefield changes the test particle slope in the horizontal plane according to

$$
\begin{aligned}
\Delta x_{\text {test }}^{\prime} \approx & -\frac{e}{\gamma m c^{2}}\left[W_{x, M}\left(z_{\text {drive }}-z_{\text {test }}\right)\right. \\
& +x_{\text {drive }} W_{x, D}\left(z_{\text {drive }}-z_{\text {test }}\right) \\
& \left.+x_{\text {test }} W_{x, Q}\left(z_{\text {drive }}-z_{\text {test }}\right)\right] .
\end{aligned}
$$

The first term is a monopole wakefield that is generated by a chamber that is not mirror symmetric; this term can cause emittance to increase. The second term is dipole wakefield that is a source of collective instabilities, since it scales with the displacement of drive electron. The third term is the source mainly of tune-shift, as it scales with the displacement of the test electron. The sum of the longitudinal and transverse impedances of the APS storage ring is shown in Fig. 1, and we note that the impedance distributions in the two transverse planes are similar to each other.

In ELEGANT, we model the electron bunches with a collection of, typically $10^{4}$ to $10^{5}$ macroparticles. Rather than compute the effect of each macroparticle on those following it using the above equations, we use the more efficient method of computing a $z$-dependent current distribution (typically with a bin size of $\sim 0.3 \mathrm{~mm}$ ), then performing convolutions of each $z$-dependent beam moment with the relevant wake function. Although it is conceptually more transparent to describe these effects in terms of the wakefields, our simulations evaluate the collective force in frequency space by multiplying the impedance with the Fourier transform of the current. This conveniently sidesteps the acausal nature of the wakefields, described above, although ELEGANT can be directed to ignore this issue even when performing computations in the time-domain.

\section{ELEGANT SIMULATION FOR KICK APERTURE}

The impedance model and methods described in the previous section have successfully reproduced various impedance-driven collective effects observed in the APS ring $[11,13,14,16]$. To further validate the simulations in a way directly relevant to phenomena observed in simulations of injection for APS-U, we employed these methods to simulate the kick aperture at various charges. The kick aperture, as the name suggests, is the aperture of the accelerator viewed as a function of the strength of a kick imparted to the beam. In this section, we describe the simulation methods and results for the kick aperture.

In addition to the impedance model and a method of using it in tracking studies, our simulations require an accurate model of the single-particle beam dynamics. As mentioned above, the starting point for the single-particle dynamics model is application of the response matrix fit method [19]. For APS, this has some challenges because of the size of the ring and the multiplicity of sources of error (e.g., quadrupole strength errors and sextupole alignment errors). Rather than attempt to determine the actual errors, which may well be impossible given the closeness of the elements and the limited number and accuracy of the beam position monitors, we instead determine a set of quadrupole strength errors and tilts that replicate the measured sameplane and cross-plane response matrices, horizontal dispersion, and vertical dispersion [20].

Because nonlinear dynamics is important in the measurements, we must perform the kick-aperture simulations 
using the same type of element-by-element tracking that would be used for determining dynamic acceptance (DA). This is different from typical collective effects simulations, in which the single-particle transport around the ring is replaced by a one-turn map, e.g., using ELEGANT's ILMATRIX element [21]. Since the simulations require a large number of simulation particles and a large number of turns, these simulations are only practical with a parallel code. Hence the parallel version of ELEGANT [22] was used throughout. The transport was modeled using symplectically-integrated kick elements, a standard feature of ELEGANT and many other codes. A fourth-order integrator [23] was used with the exact (i.e., unexpanded) Hamiltonian in the hard-edge approximation.

Synchrotron radiation is calculated in all magnets, which results in an energy loss of $5.353 \mathrm{MeV}$ per turn. Both the classical and quantum aspects of the radiation are included, so that both damping and quantum excitation are modeled. For simplicity, a single rf cavity in Sector 40 represents all 16 APS rf cavities distributed in sectors 36, 37, 38, and 40. The rf harmonic number is $h=1296$ and the total $\mathrm{rf}$ voltage is $9.5 \mathrm{MV}$.

In order to avoid spurious effects in the kick-aperture tracking, the starting particle distribution should be at equilibrium on the closed orbit that includes synchrotron radiation effects. It should also reflect the effect of the longitudinal impedance on the bunch length and energy spread. In order to generate this equilibrium particle distribution, preliminary tracking was performed without the impedance with 100000 particles for 10000 turns, which is more than sufficient to reach equilibrium given that we started with a beam distribution characterized by the nominal lattice functions and emittances. We then track another 10000 turns with the impedance, which is sufficient for the beam to reach the equilibrium bunch length and energy spread. The output from this tracking run was used as the initial distribution for all subsequent simulations. Note that although we used 100000 particles to ensure that the equilibrium properties of the beam had fully converged, the results shown below changed little even if as few as 10000 particles were used.

In order to investigate the impact of collective effects on dynamic aperture, large-amplitude beam motion is excited by a fast kicker, and the beam loss dependence on the bunch charge is simulated. The simulation results are compared to the experiment.

\section{KICK APERTURE MEASUREMENT AND ANALYSIS}

Data for the kick aperture were collected in January 2017. Beam parameters for the experiments are listed in Table I. Prior to data collection, the linear optics was characterized using the response matrix (RM) fit method $[19,20]$. The model generated by the RM fit does not reproduce measured betatron tunes and chromaticity to the
TABLE I. Main beam parameters.

\begin{tabular}{lc}
\hline \hline Parameter & Value \\
\hline Tune $\nu_{x}, \nu_{y}$ & $36.154,19.223$ \\
Emittance ratio $\epsilon_{y} / \epsilon_{x}$ & $1 \%$ \\
Chromaticity $\xi_{x}, \xi_{y}$ & $6.59,5.85$ \\
rf voltage & $9.5 \mathrm{MV}$ \\
\hline \hline
\end{tabular}

level required for benchmarking of turn by turn motion. Further tune adjustment in the model of 0.007 was needed, which constitutes a $2 \times 10^{-4}$ fraction of the overall tune, as well as chromaticity adjustment of 0.5 which corresponds to a $1 \%$ error in sextupole strength. The stable range of a single-bunch current was explored at fixed chromaticities $\xi_{x}, \xi_{y}=6.6,5.9$, for which a single bunch was found to be stable up to $5.33 \mathrm{~mA}$. Kick apertures were measured with bunch currents of $0.9,2.3$, and 4.1 , which are all $<5.33 \mathrm{~mA}$, which justifies the use of equilibrium particle distributions (Sec. III) in the simulations.

The betatron oscillations that a kick imparts on a single bunch were measured using turn-by-turn beam position monitors (BPM). Comparison of the betatron oscillations after the kick as obtained by measurement and simulation (Fig. 2) shows that decoherence is significant in the horizontal plane, and that the amplitude of beam oscillation due to coupling exceeds $0.5 \mathrm{~mm}$ in vertical plane. The decoherence in the measurement agrees reasonably well with the simulation.

Benchmarking of the turn-by-turn motion requires good knowledge of the kick amplitude. To reduce the effect of the decaying amplitude and improve the kicker calibration accuracy, data from many BPMs was used, but only from the first turn after the kick. The measured and simulated first-turn trajectories were compared, and the kick amplitude in the simulations was adjusted to minimize the difference. The fit resulted in the kicker calibration of $0.118 \mathrm{mrad} / \mathrm{kV}$. Figure 3 compares the measured and simulated trajectory after the kicker calibration. The difference between calculated and measured trajectories does not show any clear pattern, which means that it is random and likely dominated by BPM gain errors.
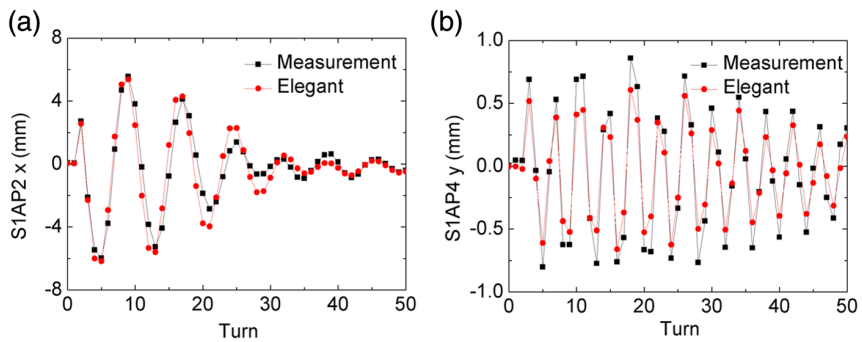

FIG. 2. Beam oscillation in horizontal (a) and vertical (b) planes following a 0.3 mrad kick in the horizontal plane for a $0.9 \mathrm{~mA}$ bunch. 


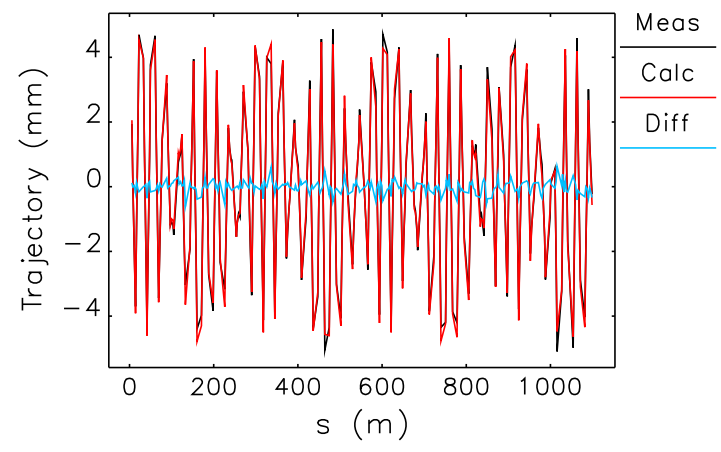

FIG. 3. Comparison of the measured and calculated first-turn trajectory after the difference was minimized by adjusting the kicker calibration.

Having calibrated the linear optics and the kicker deflection, we next performed a basic check of the nonlinear dynamics by comparing the measured and simulated tune shift with amplitude. To determine the tune shift, measured and simulated turn-by-turn beam position data were used for a series of kick amplitudes. Due to fast decoherence, taking the FFT of the beam oscillation on one BPM does not estimate the tune well. Instead, as suggested in [24], beam motion is recorded on many BPMs, the tune is determined separately for each BPM using numerical analysis of fundamental frequencies (NAFF) [25], and then the average tune is calculated by averaging over all BPMs. For a typical motion lasting 30-40 turns recorded on 270 $\mathrm{BPMs}$, this gives a standard deviation of the average tune of $1 \times 10^{-5}$. Figure 4 shows comparison of the calculated and measured tune shift with amplitude. The calculated tunes were obtained in exactly the same way as the measured tunes. The difference between measured and simulated second-order tune shift with amplitude is $7 \%$.

We next turned to comparison of the dynamic acceptance from measurements and simulations. The dynamic acceptance is the boundary in phase space beyond which particles are lost. This acceptance can be determined by kicking the beam with increasingly large amplitude until losses are observed. In our case, the losses were assessed

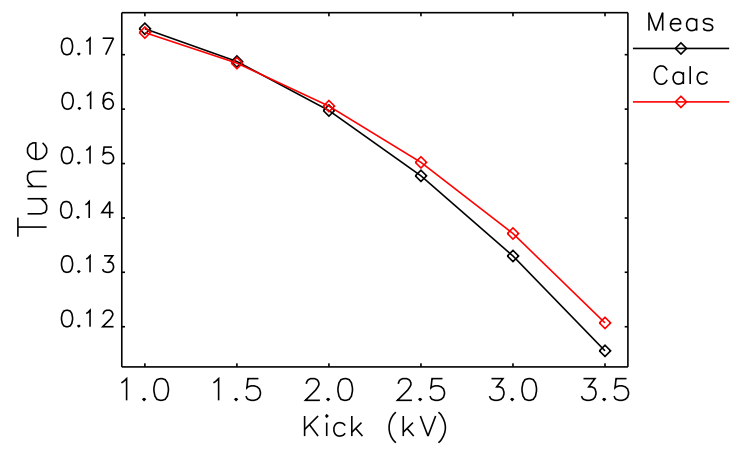

FIG. 4. Horizontal detuning with amplitude. Error bars based on averaging over 270 BPMs are not shown as they are smaller that the symbols on the plot. by measuring the bunch current with a DC Current Transformer (DCCT) before and after the kick. By taking measurements at several bunch currents $(0.9 \mathrm{~mA}, 2.3 \mathrm{~mA}$, and $4.1 \mathrm{~mA}$ ), we were able to assess the influence of collective effects and compare this to the predictions of the simulations. Figure 5 shows the measured and simulated kick apertures for different bunch currents. The simulated charge dependence of the aperture agrees very well with the measurements. One can see that the amplitude at which $50 \%$ of the beam is lost is nearly independent of bunch current and equal to $0.47 \mathrm{mrad}$ for both measurement and simulation. Also, a bunch with higher charge has more losses for kicks weaker than $0.47 \mathrm{mrad}$, but less losses for kicks stronger than $0.47 \mathrm{mrad}$. Figure 6 gives direct comparison of the dynamic acceptance for each bunch charge. The simulations underestimate the acceptance by only about $2 \%$.

An additional, even more stringent test of the agreement between the model and the experiment is the comparison of the beam losses as a function of turn number. Generally, the turn-by-turn bunch charge can be extracted from the BPM sum signal, however, the APS BPMs report logarithm of the true BPM sum signal mixed with the coordinate. To perform the comparison, the BPM sum signal was calibrated versus the bunch charge as measured by the
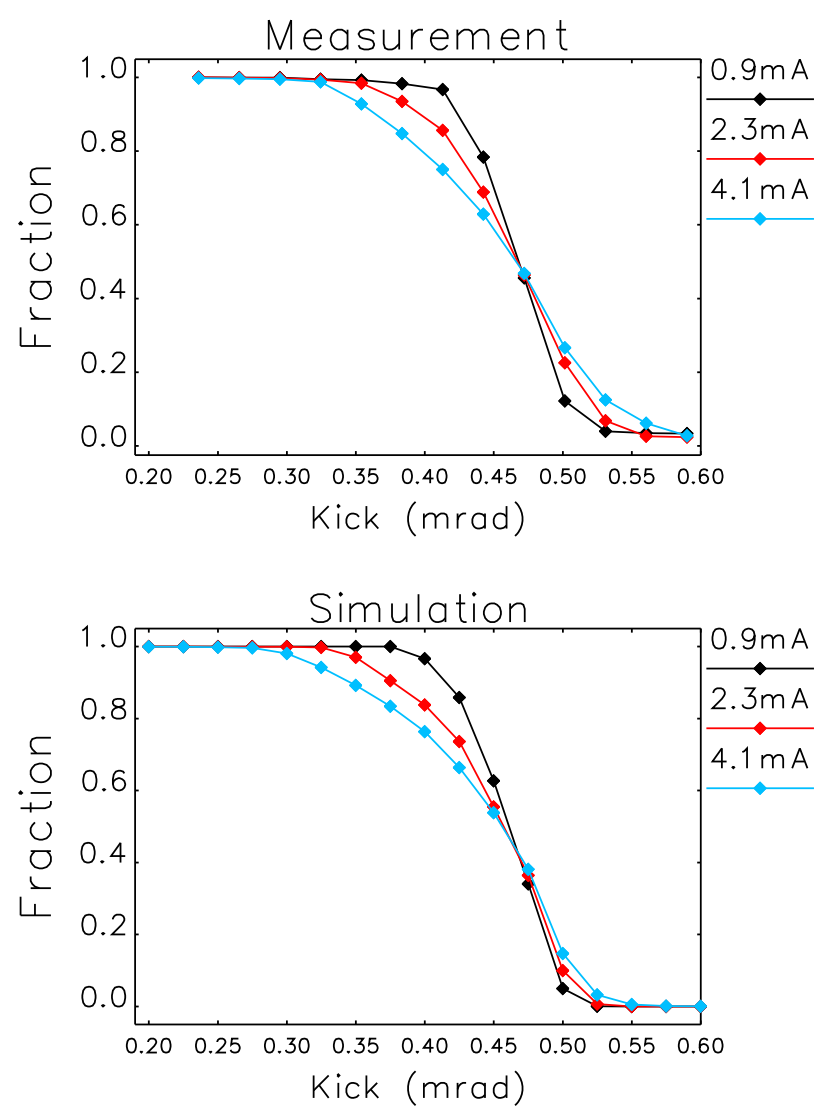

FIG. 5. Comparison of the kick aperture experiment and simulation for different bunch charge. The charge dependence agreement is remarkable. 

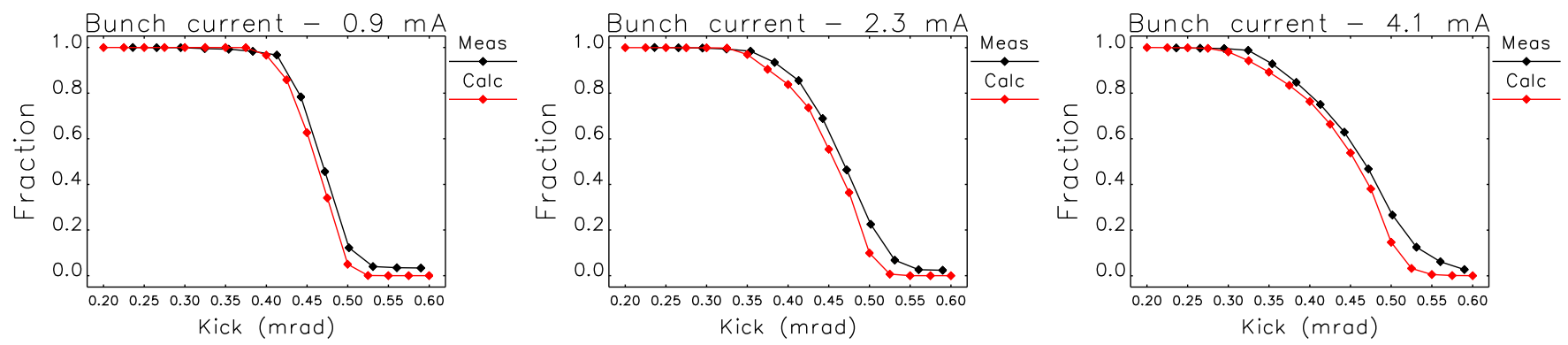

FIG. 6. Comparison of the kick aperture experiment and simulation separately for each bunch charge. The simulation underestimates the dynamic aperture by about $2 \%$. For a given kick, the simulations underestimate the beam losses by about $10 \%$.

current monitor, while the sum signal dependence on the coordinate was simply ignored (since the vertical beam motion is much smaller than the horizontal, the vertical BPM sum signal was used). Figure 7 shows comparison of the normalized bunch charge as a function of turns after the kick; lines show simulations and symbols represent measurements. While the simulations do not exactly match the experiment, they reproduce the general features, such as the speed of the losses and the steplike behavior. The discrepancy of the remaining charge after the kick approximately corresponds to the discrepancy shown in Fig. 6.
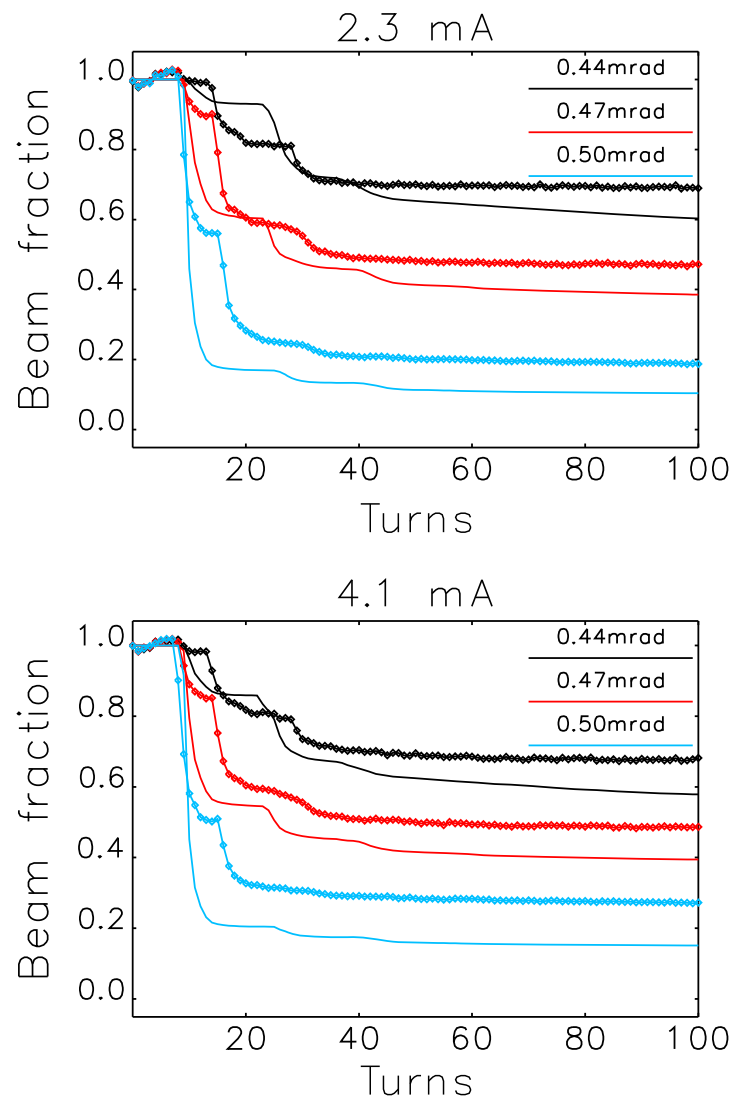

FIG. 7. Comparison of the turn-by-turn beam normalized bunch intensity for several kick amplitudes for a 2.3-mA bunch (top) and a 4.1-mA bunch (bottom). Lines show data from simulations, while connected points are from the experiments.
We can understand the basic phenomenology of the beam loss as a function of current and kick strength by considering the schematic in Fig. 8. The dynamic acceptance is represented as the black curve in the figure, and particles are lost if the kick is large enough to put them beyond the dynamic acceptance. The fraction of particles that are lost when the bunch is near the boundary will depend on the emittance of the bunch. Figure 8(a) and 8(b) show cases where the beam centroid is kicked just below and just above the aperture, respectively. As indicated in the figure, higher (lower) beam current is accompanied by larger (smaller) post-kick emittance.

We can get a more quantitative picture and understand the basic order of magnitude of the observed emittance growth by introducing a simple test particle model in the spirit of those described in [26]. For this model we consider a test electron with natural transverse frequency $\omega_{j}$ and transverse position in the bunch $z_{j}$ that is driven by the transverse wakefield of the preceding electrons. The equation of motion is

$$
\begin{aligned}
\frac{d^{2} x_{j}}{d t^{2}}+\omega_{j}^{2} x_{j} & =\frac{e^{2} N_{e}}{\gamma m c T_{0}} \int d z^{\prime} \lambda\left(z^{\prime}\right) W_{x}\left(z_{j}-z^{\prime}\right)\langle x(t)\rangle \\
& =\langle x(t)\rangle \mathcal{W}_{j},
\end{aligned}
$$

(a)

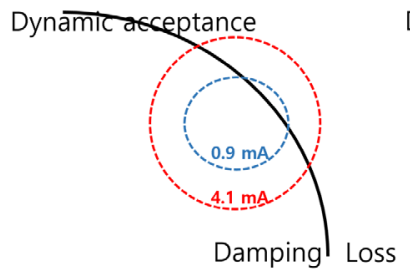

(b)

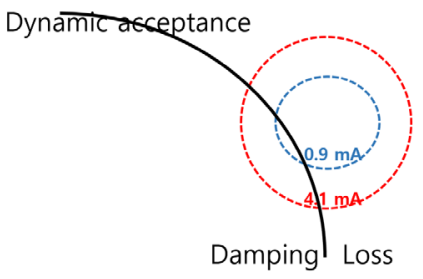

FIG. 8. Schematics for understanding current-dependence of the measured acceptance. The black curve represents the dynamic acceptance (DA), while the colored lines indicate the extent of the beam. For larger bunch current, after being kicked the beam size inflates more significantly due to collective effects. As a result, for a weaker kick (a), a larger fraction of the high-current bunch is lost; more of the low-current bunch is on the damping side of the DA boundary. Similarly, for a stronger kick (b), a larger fraction of the high-current bunch is still within the acceptance. 
where $\langle x\rangle$ is the beam dipole moment and $\mathcal{W}_{j}$ is the wakefield related force that we have defined in terms of the current distribution $\lambda(z)$ (normalized such that $\int d z \lambda(z)=1$ ), the number of electrons in the bunch $N_{e}$, and the revolution time $T_{0}$. We imagine that the entire bunch executes large-amplitude betatron oscillations after being kicked to the angle $x_{0}^{\prime}$ at $t=0$, so that

$$
\langle x(t)\rangle=\frac{c x_{0}^{\prime}}{\omega_{\beta}} \sin \left(\omega_{\beta} t\right) e^{-t / \tau}
$$

Here, we assume centroid oscillation frequency is close to that of any individual particle, $\Delta \omega_{j}=\omega_{j}-\omega_{\beta} \ll \omega_{\beta}$, while $\tau$ provides a simple damping model of the coherent betatron oscillations due to chromatic and nonlinear effects; a more accurate model of these physics is described in Ref. [27]. Since the damping of the coherent oscillation is primarily due to the spread in frequency, most particles satisfy $\left|\Delta \omega_{j}\right| \lesssim 1 / \tau$; for the APS the damping time is about 30 turns, $\tau \approx 30 T_{0}$.

Within this model, if we retain only lowest order terms in $\tau \omega_{j} \approx \tau \omega_{\beta} \gg 1$ and consider times much longer than the damping time, $t \gg \tau$, the solution to (5) and (6) with $x(0)=0$ and $x^{\prime}(0)=x_{0}^{\prime}$ is

$$
\begin{aligned}
x_{j}(t \gg \tau) \approx & \frac{c x_{0}^{\prime}}{\omega_{\beta}}\left\{1-\frac{\tau \mathcal{W}_{j}\left(\tau \Delta \omega_{j}\right)}{2 \omega_{\beta}\left[1+\left(\tau \Delta \omega_{j}\right)^{2}\right]}\right\} \cos \left(\omega_{j} t\right) \\
& -\frac{c x_{0}^{\prime}}{\omega_{\beta}} \frac{\tau \mathcal{W}_{j}}{2 \omega_{\beta}\left[1+\left(\tau \Delta \omega_{j}\right)^{2}\right]} \sin \left(\omega_{j} t\right) .
\end{aligned}
$$

The first term oscillates in phase with the main bunch and decreases due to dephasing if $\omega_{j}$ differs $\omega_{\beta}$, while the second term tends to increase the oscillation amplitude; both lead to emittance growth. For example, if we define the amplitude of the oscillation as $A_{x}$, particles that oscillate at the same frequency as the centroid get the amplitude

$$
\Delta \omega_{j}=0: A_{x}=\frac{c x_{0}^{\prime}}{\omega_{\beta}} \sqrt{1+\frac{\tau^{2} \mathcal{W}_{j}^{2}}{4 \omega_{\beta}^{2}}} .
$$

On the other hand, assuming $\mathcal{W}_{j}>0$ those particles whose frequency difference $\Delta \omega_{j}=1 / \tau\left(\Delta \omega_{j}=-1 / \tau\right)$ end up with the smallest (largest) oscillation amplitude,

$$
\begin{aligned}
& \Delta \omega_{j}=+1 / \tau: A_{x}=\frac{c x_{0}^{\prime}}{\omega_{\beta}} \sqrt{1-\frac{\tau \mathcal{W}_{j}}{2 \omega_{\beta}}+\frac{\tau^{2} \mathcal{W}_{j}^{2}}{8 \omega_{\beta}^{2}}}, \\
& \Delta \omega_{j}=-1 / \tau: A_{x}=\frac{c x_{0}^{\prime}}{\omega_{\beta}} \sqrt{1+\frac{\tau \mathcal{W}_{j}}{2 \omega_{\beta}}+\frac{\tau^{2} \mathcal{W}_{j}^{2}}{8 \omega_{\beta}^{2}}} .
\end{aligned}
$$

Equations (9) and (10) approximately give the maximum and minimum amplitude of the particles, provided $\tau \mathcal{W}_{j} / \omega_{\beta}<1$. We graph the fractional change in the oscillation amplitude of the particles as a function of the longitudinal position in Fig. 9. Panels (a), (b), and (c) assume the current of $0.9,2.3$, and $4.1 \mathrm{~mA}$, respectively, and each plot shows the amplitude for the frequency differences (8)-(10).

Within this test particle theory nearly all the particles have amplitudes that are bounded by the blue and red lines in Fig. 9. This implies, for example, that we expect the first sign of losses to appear for the $2.3 \mathrm{~mA}$ bunch at a kick amplitude that is $\sim 88 \%$ of that at $0.9 \mathrm{~mA}$, while the $4.1 \mathrm{~mA}$ bunch should have a similar sign of loss at $\sim 75 \%$ the $0.9 \mathrm{~mA}$ kick. Both of these predictions agree reasonably well with that shown in Fig. 5.

The theory just presented gives a reasonable description of the basic physics and scalings of the charge-dependent beam loss, but is only approximate. In particular, the model has only a simple description of the nonlinear and chromatic effects, neglects the synchrotron motion entirely, and is not a self-consistent description of the collective forces. To look at the dynamics in more detail we turn to the numerical ELEGANT simulations. Figure 10 compares the horizontal phase space evolution for bunches with the relatively low current of $0.9 \mathrm{~mA}$ to those of $4.1 \mathrm{~mA}$ for a kick of $0.35 \mathrm{mrad}$. One can already see on turn 12 that the phase space distribution of the $4.1 \mathrm{~mA}$ bunch becomes fatter, which then lead to some particles being kicked out of the separatrix. This result justifies the schematic descriptions in Fig. 8.
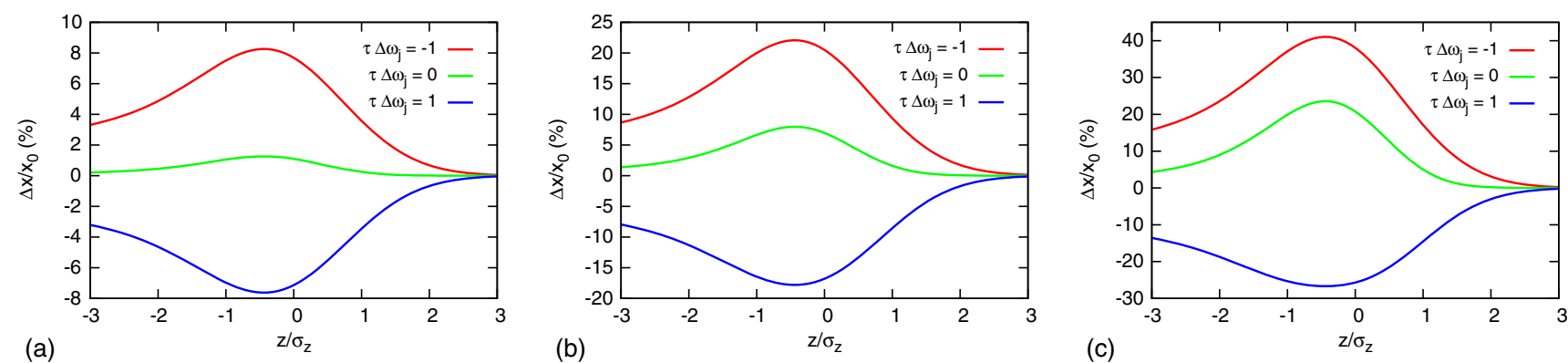

FIG. 9. Theoretical fractional change in oscillation amplitude for various frequency differences at (a) $0.9 \mathrm{~mA}$, (b) $2.3 \mathrm{~mA}$, and (c) $4.1 \mathrm{~mA}$. Within this model nearly all the particles should be between the blue and red lines at $\Delta \omega_{j}= \pm 1 / \tau$. 

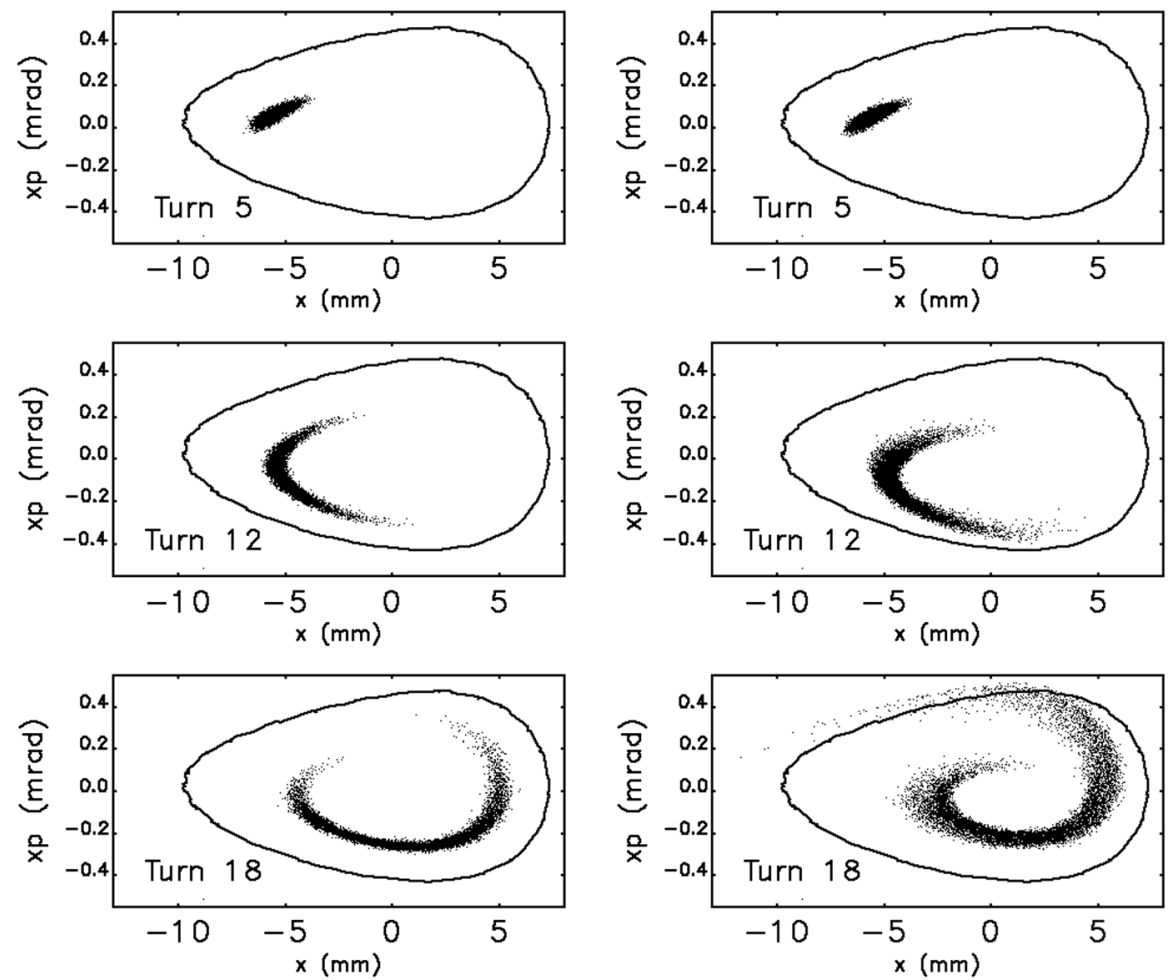

FIG. 10. Details of the horizontal phase space evolution of the $0.9 \mathrm{~mA}$ bunch (left column) and $4.1 \mathrm{~mA}$ bunch (right column). Solid line shows the separatrix of the motion.

\section{CONCLUSIONS}

We have described simulations and measurements of the impact of collective effects on dynamic acceptance in APS. Although the accurate prediction of nonlinear beam dynamics with collective effects is extremely challenging, the simulations agree well with experimental measurements. Based on the agreement of simulations with measurements, the simulation describes currentdependent nonlinear beam dynamics in detail. This result provides confidence in predictions of high-charge injectedbeam dynamics in the APS upgrade storage ring.

\section{ACKNOWLEDGMENTS}

We would like to thank L. Emery (APS) and R. Nagaoka (SOLEIL) for providing helpful information and for the many useful discussions. This research was supported by the U.S. Department of Energy, Office of Science, under Contract No. DE-AC02-06CH11357 and also by Basic Science Research Program through the National Research Foundation of Korea (NRF-2019R1A2C1004862).

[1] D. Einfeld and M. Plesko, Design of a diffraction-limited light source SPIE Proceedings, (2013), 201 (1993).

[2] MAX IV Detailed Design Report, https://www.maxiv.lu.se/ accelerators-beamlines/accelerators/accelerator-document ation/max-iv-ddr/.
[3] L. Liu, N. Milas, A. H. C. Mukai, X. R. Resende, A. R. D. Rodriguet, and F.H. Sa, in Proceedings of the 4th International Particle Accelerator Conference, IPAC2013, Shanghai, China, 2013 (JACoW, Shanghai, China, 2013), p. 1874.

[4] ESRF-EBS: The Extremely Brilliant Source Project, http:// indico.psi.ch/conferenceDisplay.py?confId=5589.

[5] APS Upgrade introduction, https://www.aps.anl.gov/ APSUpgrade.

[6] Spring-8-II conceptual design report, http://rsc.riken.jp/ eng/index.html.

[7] C. Steier et al., in Proc. of IPAC 2016 (2016) p. 2956.

[8] M. Borland, Y. P. Sun, V. Sajaev, R. R. Lindberg, and T. Berenc, Lower emittance lattice for the Advanced Photon Source upgrade using reverse bending magnets, in Proceedings of the 2016 North American Particle Accelerator Conference (2016), pp. 877-880.

[9] R. Abela, W. Joho, P. Marchand, S. V. Milton, and L. Z. Rivkin, Design considerations for a Swiss Light Source, in Proceedings of the 1992 European Particle Accelerator Conference, Berlin (1992), pp. 486-488.

[10] L. Emery and M. Borland, Possible long-term improvements to the advanced photon source, in Proceedings of the 20th Particle Accelerator Conference, PAC-2003, Portland, OR, 2003 (IEEE, New York, 2003), pp. 256-258.

[11] R. R. Lindberg and A. Blednykh, Instability thresholds for the advanced photon source multi-bend achromat upgrade, in Proceedings of IPAC 2015, Richmond, VA, USA (JACoW, 2015), p. 1822, DOI: 10.18429/JACoWIPAC2015-TUPJE077. 
[12] R. Lindberg, M. Borlnad, and A. Blednykh, Collective effects at injection for the APS-U MBA lattice, in Proceedings of NAPAC2016, Chicago, IL, USA (JACoW, 2016), p. 901, ISBN 978-3-95450-180-9.

[13] Y. Chae, Impedance database and its application to the aps storage ring, in Proceedings of the 20th Particle Accelerator Conference, PAC-2003, Portland, OR, 2003 (IEEE, New York, 2003), p. 3017.

[14] Y.-C. Chae, Impedance database ii for the advanced photon source storage ring, in Proceedings of the 22nd Particle Accelerator Conference, PAC-2007, Albuquerque, NM (IEEE, New York, 2007), p. 4336.

[15] M. Borland, ELEGANT: A flexible sdds-compliant code for accelerator simulation, Advanced Light Source Technical Report LS-287, , 2000.

[16] V. Sajaev, M. Borland, Y.-C. Chae, and L. Emery, Experimental verification of single-bunch accumulation limit dependence on impedance at the APS, in Proceedings of the 25th Particle Accelerator Conference, PAC-2013, Pasadena, CA, 2013 (IEEE, New York, 2013), p. 405.

[17] A. Piwinski, Impedances in lossy elliptical vacuum chambers, DESY Technical Report 94-068, 1994.

[18] W. Bruns, The GdfidL electromagnetic field simulator, http://www.gdfidl.de/.

[19] J. Safranek, Experimental determination of storage ring optics using orbit response measurements, Nucl. Instrum. Methods Phys. Res., Sect. A 388, 27 (1997).
[20] V. Sajaev and L. Emery, in Proceedings of the 8th European Particle Accelerator Conference, Paris, 2002 (EPS-IGA and CERN, Geneva, 2002), p. 742.

[21] M. Borland, T. Berenc, L. Emery, and R. Lindberg, Simultaneous simulation of multi-particle and multi-bunch collective effects for the APS ultra-low-emittance upgrade., in Proceedings of the 2015 International Computational Accelerator Physics Conference, Shanghai, pp. 61-65, 2015.

[22] Y. Wang and M. Borland, Pelegant: A parallel accelerator simulation code for electron generation and tracking, in Proceedings of the 12th Advanced Accelerator Concepts Workshop, Wisconsin (2006), p. 241.

[23] E. Forest, Canonical integrators as tracking codes, AIP Conf. Proc. 184, 1106 (1989).

[24] B. Podobedov et al., Single micron single-bunch turn-byturn BPM resolution achieved at NSLS-II, in Proceedings of the 2016 International Particle Accelerator Conference, Busan (2016), pp. 2095-2098.

[25] J. Lascar, C. Froeschle, and A. Celletti, The measure of chaos by the numerical analysis of fundamental frequencies. application to the standard mapping, Physica D: Nonlinear Phenomena 56, 253 (1992).

[26] A. W. Chao, Physics of Collective Beam Instabilities in High Energy Accelerators (Wiley, New York, 1993).

[27] R. E. Meller, A. W. Chao, J. M. Peterson, S. G. Peggs, and M. Furman, Decoherence of a kicked beam, Technical Report No. SSC-N-360, 1987, http://lss.fnal.gov/archive/ other/ssc/ssc-n-360.pdf. 